\title{
Value of Islet 1 and PAX8 in identifying metastatic neuroendocrine tumors of pancreatic origin
}

\author{
Jamie Koo ${ }^{1}$, Richard B Mertens ${ }^{1}$, James M Mirocha ${ }^{2}$, Hanlin L Wang ${ }^{3}$ and Deepti Dhall ${ }^{1}$ \\ ${ }^{1}$ Department of Pathology, Cedars-Sinai Medical Center, Los Angeles, CA, USA; ${ }^{2}$ Biostatistics Core, Research \\ Institute, Cedars-Sinai Medical Center, Los Angeles, CA, USA and ${ }^{3}$ Department of Pathology, University of \\ California, Los Angeles, CA, USA
}

\begin{abstract}
Neuroendocrine tumors can present as liver metastases before discovery of the primary tumor. Islet 1 and PAX8 have recently been proposed as markers for neuroendocrine tumors of pancreatic origin. In this study, we compared the utility of Islet 1 and PAX8 in distinguishing pancreatic neuroendocrine tumors from neuroendocrine tumors of other sites and determined the usefulness of an immunohistochemical panel, including TTF1, CDX2, Islet 1 and/or PAX8, in identifying metastatic pancreatic neuroendocrine tumors. A total of 110 primary neuroendocrine tumors (33 pancreatic, 31 pulmonary, 23 ileal, 14 rectal, and 9 gastric) and 73 metastatic neuroendocrine tumors (28 pancreatic, 5 pulmonary, 37 ileal, 1 rectal, 1 colonic, and 1 duodenal) were studied. Islet 1 and PAX8 were positive in $27 / 33(82 \%)$ and $29 / 33(88 \%)$, respectively, of primary pancreatic neuroendocrine tumors, and in 19/28 (68\%) and $15 / 28(54 \%)$, respectively, of metastatic pancreatic neuroendocrine tumors. No cases of primary $(0 / 23)$ or metastatic $(0 / 37)$ ileal neuroendocrine tumors were positive with either Islet 1 or PAX8. There was Islet 1 positivity in 2/31 (6\%) primary pulmonary, 12/14 (86\%) primary rectal, and 1/1 metastatic rectal neuroendocrine tumors, and PAX8 positivity in $7 / 31$ (23\%) primary pulmonary, $11 / 14(79 \%)$ primary rectal, and 2/9 (22\%) primary gastric neuroendocrine tumors. ROC curve analysis incorporating sensitivity and specificity data of immunohistochemical panels for metastatic pancreatic neuroendocrine tumors showed that a four-stain panel, including Islet 1, PAX8, TTF1, and CDX2 significantly outperformed a three-stain panel composed of PAX8, TTF1, and CDX2 $(P=0.019)$, and also showed a trend for better performance compared with a three-stain panel composed of Islet 1 , TTF1, and CDX2 $(P=0.072)$. Both Islet 1 and PAX8 are reliable immunohistochemical markers for pancreatic neuroendocrine tumors and would be useful adjuncts to other markers (TTF1, CDX2) currently used to work up a metastatic neuroendocrine tumor of unknown primary.

Modern Pathology (2012) 25, 893-901; doi:10.1038/modpathol.2012.34; published online 2 March 2012
\end{abstract}

Keywords: carcinoid; immunohistochemistry; Islet 1; metastasis of unknown primary; neuroendocrine tumor; pancreas; PAX8

Neuroendocrine tumors are derived from the diffuse neuroendocrine system and can arise from a variety of primary sites, with the most common sites including the bronchopulmonary system, gastrointestinal tract, and pancreas. ${ }^{1}$ Gastrointestinal and pancreatic neuroendocrine tumors are frequently slow growing and indolent, and may present as

Correspondence: Dr J Koo, MD, Department of Pathology, CedarsSinai Medical Center, 8700 Beverly Boulevard, Room 8709, Los Angeles, CA 90048, USA.

E-mail: jamie.koo@cshs.org

Received 10 November 2011; revised 4 January 2012; accepted 4 January 2012; published online 2 March 2012 metastases to the liver before the primary tumor has been detected. ${ }^{1}$ Identification of the primary site in a patient with metastatic neuroendocrine tumor has become increasingly important in the consideration of surgical and/or pharmacological therapies. Because neuroendocrine tumors from various sites share similar histological features, it is often not possible to predict the site of origin of a metastatic tumor based on hematoxylin and eosin (H\&E) staining alone. Immunohistochemical staining with TTF1 and CDX2 has been shown to be helpful in evaluating metastatic neuroendocrine tumors of the pulmonary and gastrointestinal origin, respectively. ${ }^{2-9}$ Until recently, no widely available or 
accepted marker for neuroendocrine tumors of pancreatic origin was available, but studies in the past few years have proposed Islet 1 and PAX8 as markers for pancreatic neuroendocrine tumors. ${ }^{10-12}$

The purpose of this study was to compare the utility of immunohistochemistry for Islet 1 and PAX8 in distinguishing both primary and metastatic neuroendocrine tumors of pancreatic origin from those of other sites, as well as to determine the usefulness of an immunohistochemical panel including TTF1, CDX2, Islet 1, and/or PAX8 in identifying the primary site of origin of metastatic neuroendocrine tumors in the liver.

\section{Materials and methods}

\section{Patients and Tumor Specimens}

Institutional review board approval was obtained for this study. A total of 183 tumor specimens from 150 patients were selected from the archives of the Cedars-Sinai Medical Center Department of Pathology from 1992 to 2011. There were 110 primary neuroendocrine tumors (33 pancreatic, 31 pulmonary, 23 ileal, 14 rectal, and 9 gastric) and 73 metastatic neuroendocrine tumors (28 pancreatic, 5 pulmonary, 37 ileal, 1 rectal, 1 colonic, and 1 duodenal). All tumors were well-differentiated based on the World Health Organization (WHO) criteria. ${ }^{13,14}$ On the basis of the current WHO classification, the 33 primary pancreatic neuroendocrine tumors included 22 grade 1 (G1) and 11 grade 2 (G2) tumors, the 31 primary pulmonary neuroendocrine tumors included 23 typical carcinoids and 8 atypical carcinoids, and the 23 primary ileal neuroendocrine tumors included 18 G1 and 5 G2 tumors. Tissues from both the primary tumor and the corresponding metastatic tumor were available for 33 patients (22 with ileal, 9 with pancreatic, and 2 with pulmonary neuroendocrine tumors). In 58 cases of metastatic neuroendocrine tumor, the site of origin was based on concurrent or prior histological evaluation of the primary tumor, and in 15 cases, the site of origin was based on welldocumented clinical history or radiological identification of a defined mass.

\section{Immunohistochemistry}

Tissue sections $(4 \mu \mathrm{m})$ were cut from paraffinembedded tissue blocks and stained with antibodies against Islet 1 (clone 40.3A4, dilution 1:20, Developmental Studies Hybridoma Bank, Iowa City, IA, USA), PAX8 (polyclonal, dilution 1:50, Proteintech, Chicago, IL, USA), TTF1 (clone 8G7G3/1, predilute, Ventana Medical Systems, Tucson, AZ, USA), and CDX2 (EPR2764y, predilute, Ventana Medical Systems). Immunohistochemical staining was performed using automated staining systems (Islet 1 on Leica Bond-Max, Leica Microsystems, Chicago, IL, USA; PAX8, TTF1, and CDX2 on Ventana Benchmark,
Ventana Medical Systems). Pretreatment was performed with on-board antigen retrieval method. External positive control tissues used included normal pancreatic tissue for Islet 1, non-neoplastic kidney tissue for PAX8, non-neoplastic lung tissue for TTF1, and non-neoplastic large intestinal tissue for CDX2.

Nuclear immunoreactivity for Islet 1, PAX8, TTF1, and CDX2 was evaluated by two investigators (JK and DD). Using a scale similar to that used in prior studies, ${ }^{5,8-11}$ the extent of positive staining was semiquantitatively assessed as $0=<5 \%$ staining, $1+=5-25 \%$ staining, $2+=26-50 \%$ staining, $3+$ $=51-75 \%$ staining, and $4+=>75 \%$ staining. The intensity of nuclear staining was evaluated as weak, moderate, or strong, based on comparison with staining of external positive controls or internal positive controls if present. Tumors showing moderate to strong nuclear staining of at least $5 \%$ of cells were considered positive. In those tumors exhibiting only weak staining, the threshold for positivity was increased to at least $10 \%$ of tumor cells in an effort to avoid interpreting non-specific staining as positive. Cytoplasmic staining in the absence of nuclear staining was scored as negative.

\section{Statistical Analysis}

In the analysis of our data, we found that excluding cases with weak staining resulted in only a minimal increase in specificity, at the cost of significant loss of sensitivity. Therefore, we decided to interpret those cases with weak staining of a least $10 \%$ of cells as positive. The Fisher's exact test was used to assess differences in categorical outcomes between independent groups. The McNemar's test for related proportions was used to assess differences in sensitivity and specificity between two methods (individual stains or stain panels) in the same group. Receiver operating characteristic (ROC) curves were generated, plotting the sensitivity vs 1 - specificity. The performance of stain panels was compared using the area under the ROC curve, implementing the nonparametric method of DeLong et al. ${ }^{15}$ SAS version 9.2 (SAS Institute, Cary, NC, USA) was used for statistical calculations. The $5 \%$ significance level was used throughout.

\section{Results}

The immunohistochemistry results for primary neuroendocrine tumors are summarized in Table 1, and results for metastatic neuroendocrine tumors are summarized in Table 2. A more detailed comparison of Islet 1 and PAX8 immunohistochemistry is summarized in Table 3.

\section{Islet 1 Immunohistochemistry}

In primary pancreatic neuroendocrine tumors, nuclear staining with Islet 1 was seen in $82 \%(27 / 33)$ of cases, with the majority of cases showing $3+$ to $4+$ 
and moderate to strong staining (Figure 1). Interestingly, Islet 1 expression was also seen in a significant number of primary rectal neuroendocrine tumors $(12 / 14,86 \%)$, with $3+$ to $4+$ and strong staining present in most cases (Figure 1). Islet 1 immunoreactivity was also unexpectedly seen in a small number of primary pulmonary neuroendocrine tumors $(2 / 31,6 \%)$, both of which were classified as atypical carcinoids and were negative for TTF1 staining. All 23 primary ileal neuroendocrine tumors were negative for Islet 1 staining. In primary gastric neuroendocrine tumors, a variety of staining patterns was appreciated, including cytoplasmic staining of

Table 1 Immunoreactivity of Islet 1, PAX8, TTF1, and CDX2 in primary neuroendocrine tumors

\begin{tabular}{lrcccc}
\hline Site & $\mathrm{n}$ & Islet 1 (\%) & PAX8 (\%) & TTF1 (\%) & CDX2 (\%) \\
\hline Pancreas & 33 & $27(82)$ & $29(88)$ & 0 & 0 \\
Pulmonary & 31 & $2(6)$ & $7(23)$ & $16(52)$ & 0 \\
Ileum & 23 & 0 & 0 & 0 & $20(87)$ \\
Rectum & 14 & $12(86)$ & $11(79)$ & 0 & 0 \\
Stomach & 9 & 0 & $2(22)$ & 0 & 0 \\
\hline
\end{tabular}

Table 2 Immunoreactivity of Islet 1, PAX8, TTF1, and CDX2 in metastatic neuroendocrine tumors

\begin{tabular}{lrcccc}
\hline Primary site & $\mathrm{n}$ & Islet 1 (\%) & PAX8 (\%) & TTF1 (\%) & CDX2 (\%) \\
\hline Pancreas & 28 & $19(68)$ & $15(54)$ & 0 & $1(4)$ \\
Pulmonary & 5 & 0 & 0 & $1(20)$ & 0 \\
Ileum & 37 & 0 & 0 & 0 & $33(89)$ \\
Rectum & 1 & $1(100)$ & 0 & 0 & 0 \\
Colon & 1 & 0 & 0 & 0 & $1(100)$ \\
Duodenum & 1 & 0 & 0 & 0 & 0 \\
\hline
\end{tabular}

tumor cells, cytoplasmic staining of non-neoplastic oxyntic mucosa, nuclear staining of non-neoplastic neuroendocrine cells, and nuclear staining of adjacent stromal cells; however, as nuclear immunoreactivity of tumor cells was not observed, all nine of these cases were interpreted as negative for Islet 1.

In metastatic pancreatic neuroendocrine tumors, nuclear staining with Islet 1 was seen in $68 \%$ (19/28) of cases, with the majority of cases showing $3+$ to $4+$ and moderate to strong staining (Figure 2). Additionally, one metastatic rectal neuroendocrine tumor showed strong and diffuse immunoreactivity with Islet 1. None of the Islet 1-expressing primary or metastatic rectal neuroendocrine tumors showed co-expression of CDX2. All of the 5 metastatic pulmonary neuroendocrine tumors and the remaining 39 metastatic gastrointestinal neuroendocrine tumors were negative for Islet 1 (Figure 2).

Overall, Islet 1 had a sensitivity of $82 \%$ and a specificity of $82 \%$ for primary pancreatic neuroendocrine tumors. For metastatic pancreatic neuroendocrine tumors, Islet 1 had a sensitivity of $68 \%$ and a specificity of $98 \%$.

\section{PAX8 Immunohistochemistry}

In primary pancreatic neuroendocrine tumors, nuclear staining with PAX8 was seen in $88 \%$ (29/33) of cases, with the majority of cases showing $3+$ to $4+$ and moderate to strong staining (Figure 1). Like Islet 1, PAX8 expression was also seen in a significant number of primary rectal neuroendocrine tumors $(11 / 14,79 \%)$, with both $3+$ to $4+$ and strong staining present in many cases (Figure 1); none of the PAX8expressing primary rectal neuroendocrine tumors showed coexpression of CDX2. PAX8 nuclear immunoreactivity was also seen in 7 of 31 primary

Table 3 Comparison of Islet 1 and PAX8 immunoreactivity in neuroendocrine tumors

\begin{tabular}{|c|c|c|c|c|c|c|c|c|c|c|c|c|c|}
\hline \multirow[t]{3}{*}{ Site } & \multirow[t]{3}{*}{$\mathrm{n}$} & \multicolumn{6}{|c|}{ Islet 1} & \multicolumn{6}{|c|}{$P A X 8$} \\
\hline & & \multirow[t]{2}{*}{$\begin{array}{l}\text { Total number } \\
\text { pos }(\%)\end{array}$} & \multicolumn{4}{|c|}{$\begin{array}{l}\text { Extent of } \\
\text { staining }\end{array}$} & \multirow[t]{2}{*}{$\begin{array}{c}\text { Mod to strong } \\
\text { staining ( } \% \text { of pos) }\end{array}$} & \multirow[t]{2}{*}{$\begin{array}{l}\text { Total number } \\
\text { pos }(\%)\end{array}$} & \multicolumn{4}{|c|}{$\begin{array}{l}\text { Extent of } \\
\text { staining }\end{array}$} & \multirow[t]{2}{*}{$\begin{array}{c}\text { Mod to strong } \\
\text { staining (\% of pos) }\end{array}$} \\
\hline & & & $4+$ & $3+$ & $2+$ & $1+$ & & & $4+$ & $3+$ & $2+$ & $1+$ & \\
\hline Pancreas & 33 & $27(82)$ & 24 & 2 & 0 & 1 & $26(96)$ & $29(88)$ & 20 & 4 & 2 & 3 & $23(79)$ \\
\hline Pulmonary & 31 & $2(6)$ & 1 & 1 & 0 & 0 & $1(50)$ & $7(23)$ & 2 & 1 & 0 & 4 & $5(71)$ \\
\hline Ileum & 23 & 0 & 0 & 0 & 0 & 0 & 0 & 0 & 0 & 0 & 0 & 0 & 0 \\
\hline Rectum & 14 & $12(86)$ & 11 & 1 & 0 & 0 & $12(100)$ & $11(79)$ & 10 & 1 & 0 & 0 & $10(90)$ \\
\hline Stomach & 9 & 0 & 0 & 0 & 0 & 0 & 0 & $2(22)$ & 0 & 1 & 1 & 0 & $1(50)$ \\
\hline \multicolumn{14}{|c|}{ Liver metastases } \\
\hline Pancreas & 28 & $19(68)$ & 9 & 6 & 1 & 3 & 19 (100) & $15(54)$ & 7 & 5 & 1 & 2 & $12(80)$ \\
\hline Pulmonary & 5 & 0 & 0 & 0 & 0 & 0 & 0 & 0 & 0 & 0 & 0 & 0 & 0 \\
\hline Ileum & 37 & 0 & 0 & 0 & 0 & 0 & 0 & 0 & 0 & 0 & 0 & 0 & 0 \\
\hline Rectum & 1 & $1(100)$ & 1 & 0 & 0 & 0 & $1(100)$ & 0 & 0 & 0 & 0 & 0 & 0 \\
\hline Colon & 1 & 0 & 0 & 0 & 0 & 0 & 0 & 0 & 0 & 0 & 0 & 0 & 0 \\
\hline Duodenum & 1 & 0 & 0 & 0 & 0 & 0 & 0 & 0 & 0 & 0 & 0 & 0 & 0 \\
\hline
\end{tabular}

Extent of staining scored as follows: $1+, 5-25 \%$ staining; $2+, 26-50 \%$ staining; $3+, 51-75 \%$ staining; and $4+,>75 \%$ staining. 

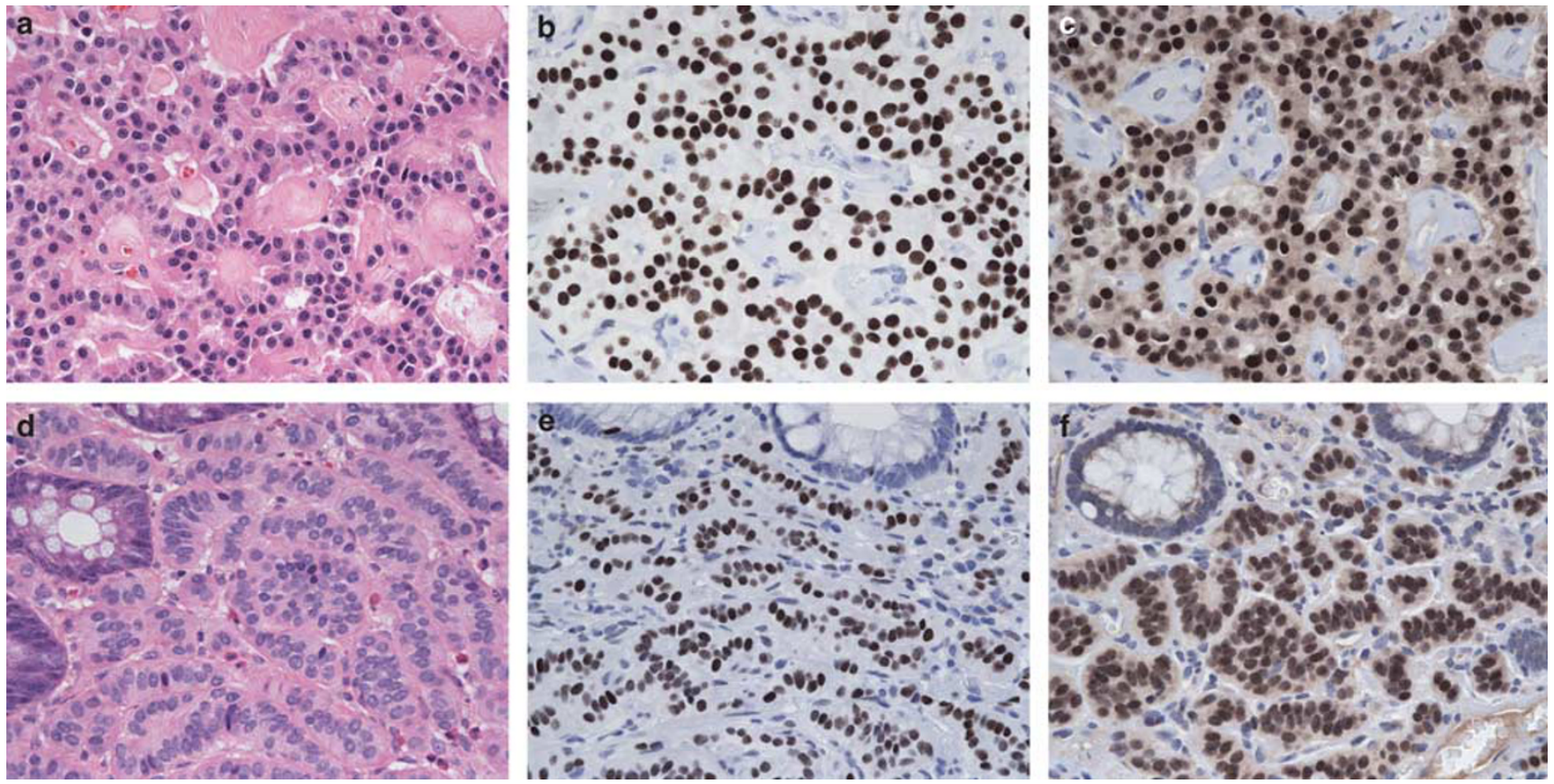

Figure 1 Islet 1 and PAX8 immunohistochemical staining in primary pancreatic neuroendocrine tumor and primary rectal neuroendocrine tumor. (a) Primary pancreatic neuroendocrine tumor (hematoxylin and eosin (H\&E), $\times 400$ ), showing strong, $4+$ staining with Islet $1(\mathbf{b} ; \times 400)$ and strong, $4+$ staining with PAX8 $(\mathbf{c} ; \times 400)$. (d) Primary rectal neuroendocrine tumor $(\mathrm{H} \& \mathrm{E}$, $\times 400)$, showing strong, $4+$ staining with Islet $1(\mathbf{e} ; \times 400)$ and strong, $4+$ staining with PAX8 $(\mathbf{f} ; \times 400)$.
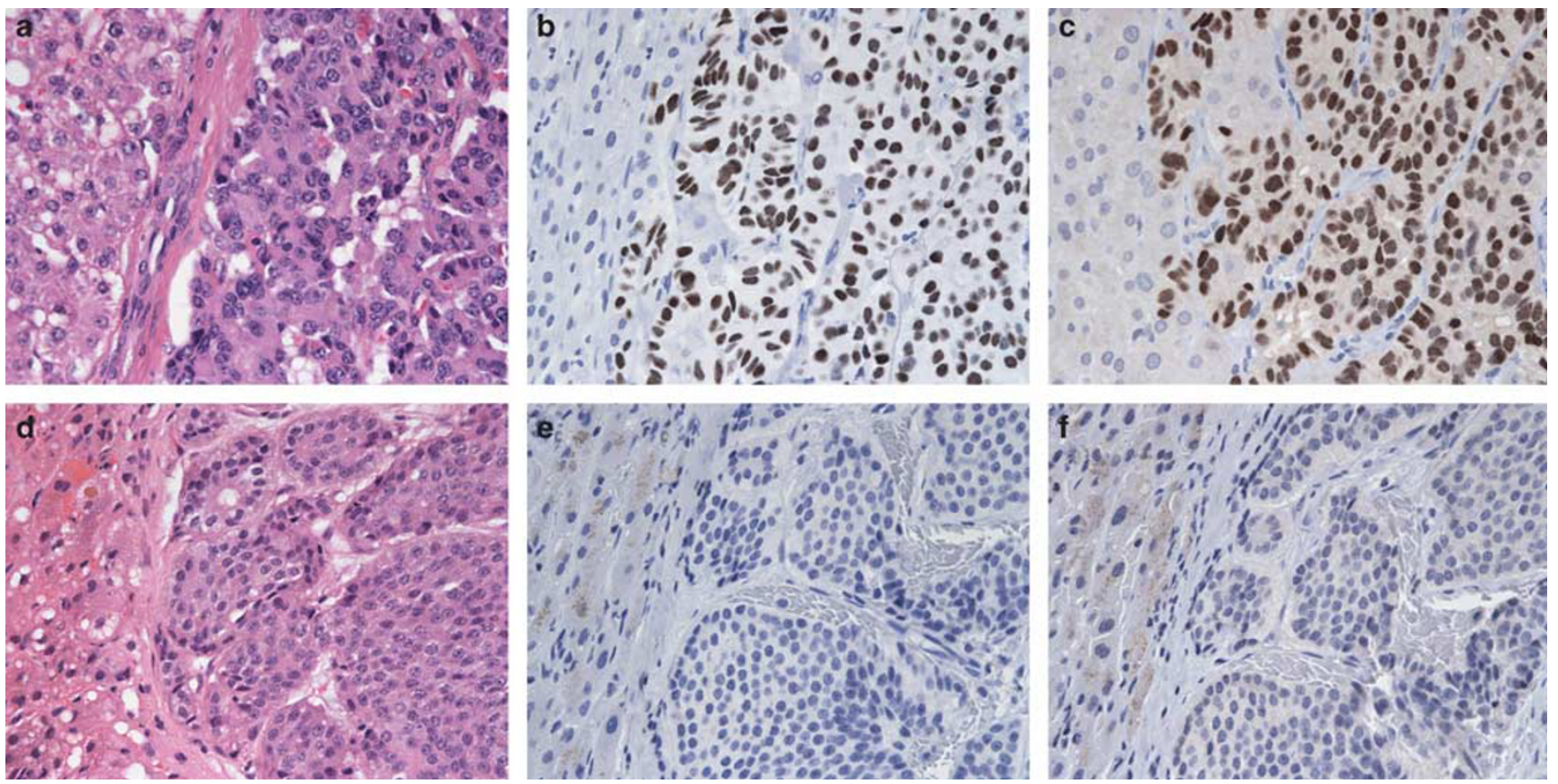

Figure 2 Islet 1 and PAX8 immunohistochemical staining in metastatic pancreatic neuroendocrine tumor and metastatic ileal neuroendocrine tumor. (a) Metastatic pancreatic neuroendocrine tumor (hematoxylin and eosin (H\&E), $\times 400$ ), showing strong, $4+$ staining with Islet $1(\mathbf{b} ; \times 400)$ and strong, $4+$ staining with PAX8 $(\mathbf{c} ; \times 400)$. (d) Metastatic ileal neuroendocrine tumor $(\mathrm{H} \& \mathrm{E}, \times 400)$, negative with Islet $1(\mathbf{e} ; \times 400)$ and negative with PAX8 $(\mathbf{f} ; \times 400)$.

pulmonary neuroendocrine tumors $(23 \%)$, with 3 cases showing $3+$ to $4+$ staining, and 5 cases showing moderate to strong staining intensity; of these 7 tumors, 5 showed variable coexpression of TTF1. Two of nine primary gastric neuroendocrine tumors demonstrated nuclear staining with PAX8, 
which was $2+$ to $3+$ and mostly weak in intensity. All 23 primary ileal neuroendocrine tumors were negative for PAX8 staining. Cytoplasmic staining without nuclear staining was observed in 10\% (11/ 110) of all primary pancreatic, gastrointestinal, and pulmonary neuroendocrine tumors, including in $19 \%(6 / 31)$ of pulmonary neuroendocrine tumors (which all showed a perinuclear dot-like pattern), $12 \%(4 / 33)$ of pancreatic neuroendocrine tumors, and $7 \%(1 / 14)$ of rectal neuroendocrine tumors.

In metastatic pancreatic neuroendocrine tumors, nuclear staining with PAX8 was seen in 54\% (15/28) of cases, with the majority of cases showing $3+$ to $4+$ and moderate to strong staining (Figure 2). None of the metastatic pulmonary or gastrointestinal neuroendocrine tumors showed PAX8 positivity. Cytoplasmic staining without nuclear reactivity was present in $21 \%(6 / 28)$ of metastatic pancreatic neuroendocrine tumors, but was not seen in metastatic tumors from other sites.

Overall, PAX8 had a sensitivity of $88 \%$ and a specificity of $74 \%$ for primary pancreatic neuroendocrine tumors. For metastatic pancreatic neuroendocrine tumors, PAX8 had a sensitivity of $54 \%$ and a specificity of $100 \%$.

\section{TTF1 Immunohistochemistry}

In primary pulmonary neuroendocrine tumors, nuclear staining with TTF1 was seen in $52 \%$ (16/31) of cases, with the majority of positive cases showing $3+$ to $4+$ staining $(13 / 16,81 \%)$. Of the 16 positively stained cases, 6 exhibited moderate to strong staining intensity. None of the primary pancreatic, ileal, rectal, or gastric neuroendocrine tumors showed immunoreactivity with TTF1. Of the five metastatic pulmonary neuroendocrine tumors in our series, nuclear staining with TTF1 was seen in one case, with strong $4+$ staining. All of the metastatic pancreatic and gastrointestinal neuroendocrine tumors were negative for TTF1.

Overall, TTF1 had a sensitivity of $52 \%$ and a specificity of $100 \%$ for primary pulmonary neuroendocrine tumors. For metastatic pulmonary neuroendocrine tumors, TTF1 had a sensitivity of $20 \%$ and a specificity of $100 \%$.

\section{CDX2 Immunohistochemistry}

In primary ileal neuroendocrine tumors, nuclear staining with CDX2 was seen in $87 \%(20 / 23)$ of cases, with the majority of the positive cases showing $3+$ to $4+$ staining $(13 / 20,65 \%)$. Of the 20 positively stained cases, $14(70 \%)$ exhibited moderate to strong staining intensity. None of the primary pancreatic, pulmonary, rectal, or gastric neuroendocrine tumors showed immunoreactivity with CDX2. In metastatic ileal neuroendocrine tumors, nuclear staining with CDX2 was seen in $89 \%(33 / 37)$ of cases, with the majority of positive
Table 4 Concordance of Islet 1 and PAX8 immunoreactivity in pancreatic neuroendocrine tumors

\begin{tabular}{lccccc}
\hline Pancreas tumors & $\mathrm{n}$ & $\begin{array}{c}\text { Islet 1+ } \\
\text { PAX8+ }\end{array}$ & $\begin{array}{c}\text { Islet 1+ } \\
\text { PAX8- }\end{array}$ & $\begin{array}{c}\text { Islet 1- } \\
\text { PAX8+ }\end{array}$ & $\begin{array}{c}\text { Islet 1- } \\
\text { PAX8- }\end{array}$ \\
\hline Primary & 33 & 25 & 2 & 4 & 2 \\
Metastatic & 28 & 12 & 7 & 3 & 6 \\
\hline
\end{tabular}

cases showing $3+$ to $4+$ staining $(27 / 33,82 \%)$. Of the 33 positively stained cases, $25(76 \%)$ demonstrated moderate to strong staining intensity. Strong, $4+$ CDX2 nuclear reactivity was also seen in one case of metastatic colonic neuroendocrine tumor. Additionally, one case of metastatic pancreatic neuroendocrine tumor showed $3+$, moderate staining with CDX2; this tumor co-expressed Islet 1 . The remaining metastatic pancreatic, pulmonary, rectal, and duodenal neuroendocrine tumors were negative for CDX2.

Overall, CDX2 had a sensitivity of $87 \%$ and a specificity of $100 \%$ for primary ileal neuroendocrine tumors. For metastatic ileal neuroendocrine tumors, CDX2 had a sensitivity of $89 \%$ and a specificity of $94 \%$.

\section{Comparison of Islet 1 and PAX8 Immunohisto- chemistry in Pancreatic Neuroendocrine Tumors}

When the sensitivities of Islet 1 and PAX8 for pancreatic neuroendocrine tumors were compared, no significant difference was found for either primary neuroendocrine tumors (82 vs $88 \%$, respectively; $P=0.69)$ or metastatic neuroendocrine tumors (68 vs $54 \%$, respectively; $P=0.34$ ). Similarly, there was no significant difference when the specificities of Islet 1 and PAX8 were compared for either primary pancreatic neuroendocrine tumors (82 vs $74 \%$, respectively; $P=0.146$ ) or metastatic pancreatic neuroendocrine tumors (98 vs 100\%, respectively; $P>0.99$ ). Although results of staining for Islet 1 and PAX8 were concordant in the majority of pancreatic neuroendocrine tumors, discordant staining was observed in 6 of 33 primary tumors and in 10 of 28 metastatic tumors, as summarized in Table 4.

\section{Staining with an Immunohistochemical Panel}

The sensitivity and specificity of several idealized immunophenotypes for identifying tumors of pancreatic, ileal, or pulmonary origin are shown in Table 5. Use of a panel with Islet 1, TTF1, and CDX2 correctly identified the primary site in $71 \%(52 / 73)$ of metastatic cases, including $64 \%(18 / 28)$ of all metastatic pancreatic neuroendocrine tumors and $89 \%$ (33/37) of all metastatic ileal neuroendocrine tumors. Similarly, use of a panel with PAX8, TTF1, and CDX2 correctly identified the primary site in 
Table 5 Use of a panel of immunohistochemical stains, including Islet 1, PAX8, TTF1, and CDX2 in predicting site of origin in primary and metastatic neuroendocrine tumors

\begin{tabular}{|c|c|c|c|c|c|}
\hline \multirow[t]{2}{*}{ Site } & \multirow[t]{2}{*}{ Pattern of immunoreactivity } & \multicolumn{2}{|c|}{ Primary tumors } & \multicolumn{2}{|c|}{ Metastatic tumors } \\
\hline & & Sensitivity (\%) & Specificity (\%) & Sensitivity (\%) & Specificity $(\%)$ \\
\hline Pancreas & Islet 1+, TTF1-, CDX2- & $27 / 33(82)$ & $63 / 77(82)$ & $18 / 28(64)$ & $44 / 45(98)$ \\
\hline Pancreas & PAX8+, TTF1-, CDX2- & $29 / 33(88)$ & $62 / 77(81)$ & $15 / 28(54)$ & $45 / 45(100)$ \\
\hline Pancreas & Islet $1+$ or PAX $8+$, TTF $1-$, CDX2- & $31 / 33(94)$ & $59 / 77(77)$ & $21 / 28(75)$ & $44 / 45(98)$ \\
\hline Ileum & CDX2+, Islet 1-, PAX8-, TTF1- & $20 / 23(87)$ & 87/87 (100) & 33/37 (89) & $35 / 36(97)$ \\
\hline Pulmonary & TTF1+, Islet 1-, PAX8-, CDX2- & $10 / 31(32)$ & 79/79 (100) & $1 / 5(20)$ & $68 / 68(100)$ \\
\hline
\end{tabular}

$67 \%(49 / 73)$ of the metastatic cases, including $54 \%$ $(15 / 28)$ of all metastatic pancreatic neuroendocrine tumors and $89 \%(33 / 37)$ of all metastatic ileal neuroendocrine tumors. There was no significant difference in the correct identification of the pancreatic site of origin of a metastatic neuroendocrine tumor when a three-stain panel consisting of Islet 1, TTF1, and CDX2 was compared with a three-stain panel composed of PAX8, TTF1, and CDX2 $(P=0.51)$.

However, when a four-stain panel consisting of Islet 1, PAX8, TTF1 and CDX2 was used, it correctly identified the primary site in $75 \%(55 / 73)$ of metastatic cases, including $75 \%(21 / 28)$ of all metastatic pancreatic neuroendocrine tumors, which was significantly better than a three-stain panel composed of PAX8, TTF1 and CDX2 $(P=0.031)$. The four-stain panel was also slightly better for determining the pancreatic site of origin of a metastatic neuroendocrine tumor when compared with a three-stain panel composed of Islet 1, TTF1, and CDX2, although this difference did not reach statistical significance $(P=0.25)$. ROC curve analysis (Figure 3) also showed that the four-stain panel significantly outperformed a three-stain panel composed of PAX8, TTF1, and CDX2 (area under curve 0.864 vs 0.768 , respectively; $P=0.019$ ), and that the four-stain panel showed a trend for better performance compared with a three-stain panel composed of Islet 1, TTF1, and CDX2 (area under curve 0.864 vs 0.810 , respectively; $P=0.072$ ).

\section{Comparison of Primary Tumors with their Corresponding Metastases}

A total of $28 / 33$ (85\%) matched primary and metastatic neuroendocrine tumor pairs showed concordant immunohistochemical staining patterns with Islet 1, PAX8, TTF1, and CDX2 (20/22 ileal, 1/2 pulmonary, and $7 / 9$ pancreatic), and there were 5 matched primary and metastatic tumor pairs with discordant staining patterns. Both the discordant pairs of ileal neuroendocrine tumor showed negative CDX2 staining in primary tumors, but $2+$ weak positivity in liver metastases (all other stains were negative). In the discordant pulmonary neuroendocrine tumor pair, the primary tumor was $3+$, weakly

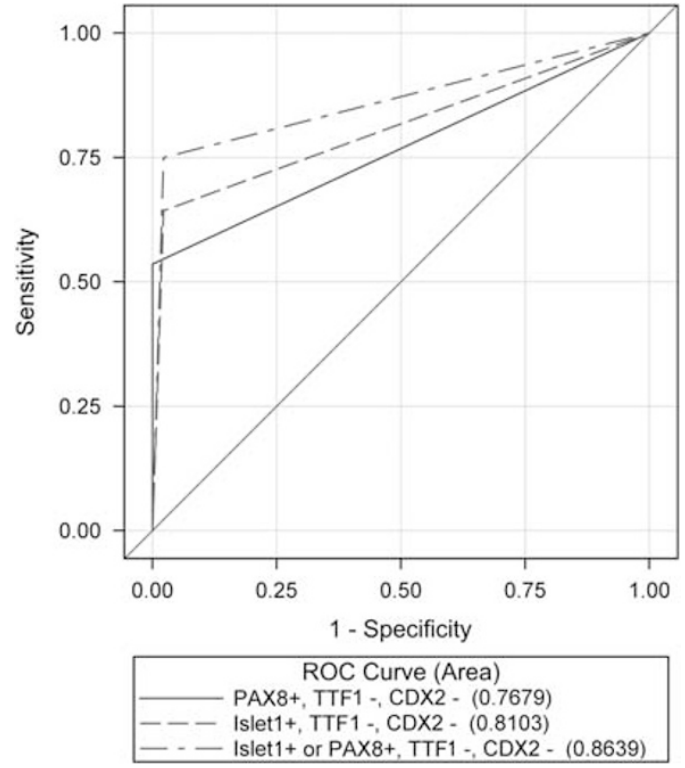

Figure 3 Receiver operating characteristic (ROC) curve analysis incorporating sensitivity and specificity data of various immunohistochemical panels, including TTF1, CDX2, Islet 1 and/or PAX8 for metastatic pancreatic neuroendocrine tumors. Areas under the curve are shown in parentheses. Please refer to text in results section for comparisons of areas under the curve between panels and corresponding $P$-values.

positive for TTF1, whereas the liver metastasis was negative for TTF1 (all other stains were negative). In one pancreatic neuroendocrine tumor pair, the primary tumor demonstrated $1+$, moderate immunoreactivity with Islet 1 , whereas the liver metastasis was negative for Islet 1 (all other stains were negative). In another pancreatic neuroendocrine tumor pair, the primary tumor showed $1+$, moderate staining with PAX8 only, whereas the liver metastasis had $2+$, moderate staining with CDX2 and $1+$, moderate staining with Islet 1 , but no PAX8 immunoreactivity (patient was status post chemotherapy for unrelated rectal adenocarcinoma).

\section{Discussion}

Neuroendocrine tumors are a diverse group of tumors arising from the diffuse neuroendocrine 
system, and thus have a wide anatomical distribution, with the most common sites including the bronchopulmonary system, gastrointestinal tract, and pancreas. Analysis of data from the Surveillance, Epidemiology, and End Results database has shown that their incidence and prevalence have increased over time. ${ }^{16}$ As they are often slow growing and nonfunctional, gastrointestinal and pancreatic neuroendocrine tumors in particular may present as metastases to the liver before the primary tumor has been detected. ${ }^{1}$

The management of metastatic neuroendocrine disease hinges critically on identification of the primary tumor source. The results of recent drug trials suggest that neuroendocrine tumors from different sites, particularly those from the pancreas, have different tumor biology and may respond differently to chemotherapeutic agents. ${ }^{17}$ Additionally, surgery can be of benefit in cases of small intestinal neuroendocrine tumor to avoid potential life-threatening complications such as bowel obstruction or ischemic bowel secondary to mesenteric fibrosis. ${ }^{18}$ Resection of the primary tumor may also result in better progression-free survival and overall survival even in patients with metastatic disease. ${ }^{19}$ Despite the availability of various imaging modalities, the primary tumor is not identified in $20-50 \%$ of gastroenteropancreatic neuroendocrine tumors. ${ }^{1}$

Histological evaluation of a liver biopsy containing metastatic neuroendocrine tumor provides another possible method for identification of the primary site of origin of the tumor. Unfortunately, neuroendocrine tumors share similar histological features regardless of their site of origin, and the primary site cannot be readily determined on routine H\&E staining. Although TTF1 and CDX2 are well established as immunohistochemical markers for metastatic neuroendocrine tumors of pulmonary and gastrointestinal origins, respectively, only recently have Islet 1 and PAX8 been proposed as immunohistochemical markers for neuroendocrine tumors of pancreatic origin. Islet 1 is a transcription factor encoded by a homeoboxcontaining gene in the LIM-homeodomain subfamily and is crucial for pancreatic and motor neuron development in mammals. ${ }^{20}$ It appears to be required for the induction of the pancreas and subsequently for the generation of endocrine islet cells. PAX8 is a transcription factor encoded by a homeobox-containing gene belonging to the $P A X$ family, which regulates organogenesis in various sites. ${ }^{21}$ Although recent studies have reported on the utility of Islet 1 and PAX8 in identifying neuroendocrine tumors of pancreatic origin, ${ }^{10-12}$ no previous studies comparing the efficacy of Islet 1 and PAX8 in this regard have been reported in the literature.

In the current study, we have demonstrated that both Islet 1 and PAX8 are highly expressed in both primary and metastatic pancreatic neuroendocrine tumors, and the frequency of expression in pancreatic neuroendocrine tumors is significantly higher than in neuroendocrine tumors of nonpancreatic origin $(P<0.0001)$. Importantly, neither Islet 1 nor PAX8 staining was present in primary or metastatic ileal neuroendocrine tumors; hence, in our study, positive staining for either Islet 1 or PAX8 clearly differentiated between neuroendocrine tumors of the pancreas and ileum, which are the two main considerations when evaluating a metastatic neuroendocrine tumor in the liver. Aberrant Islet 1 staining was seen in a small number of pulmonary neuroendocrine tumors and in the majority of rectal neuroendocrine tumors, and aberrant PAX8 positivity was present in a small number of gastric neuroendocrine tumors, in several pulmonary neuroendocrine tumors, and in the majority of rectal neuroendocrine tumors, but aberrant staining for both Islet 1 and PAX8 was observed only in cases of rectal neuroendocrine tumor. Although there was no significant difference between Islet 1 and PAX8 when comparing their individual sensitivities and specificities for pancreatic neuroendocrine tumors, there were 6/33 primary and 10/28 metastatic pancreatic neuroendocrine tumors which showed discordant staining between Islet 1 and PAX 8, suggesting that the stains may complement each other when used together as part of an immunohistochemical panel. In fact, results of an immunohistochemical panel in which both Islet 1 and PAX8 were used in conjunction with TTF1 and CDX2 indicated that use of all four stains provides a benefit over other three-stain combinations using either Islet 1 or PAX8.

A comparison of our findings on Islet 1 and PAX8 with data from previously published studies is summarized in Table 6. In the recent study by Schmitt et al, ${ }^{12}$ immunohistochemistry for Islet 1 was reported to be useful in differentiating both primary and metastatic pancreatic neuroendocrine tumors from tumors of other primary sites of origin. The authors reported positivity of Islet 1 in $69 \%$ of primary pancreatic neuroendocrine tumors and $67 \%$ of metastatic pancreatic neuroendocrine tumors, relatively similar to our corresponding findings (82 and $68 \%$, respectively). As in the study by Schmitt et $a l,{ }^{12}$ we also found Islet 1 positivity in a small number of primary pulmonary neuroendocrine tumors (2/31). Interestingly, many primary rectal neuroendocrine tumors were positive with Islet 1 in our study, an observation that has not been previously described.

Immunohistochemical staining with PAX8 was demonstrated to be effective in differentiating pancreatic neuroendocrine tumors from those of other sites in the recent studies by Long et $a 1^{10}$ and Sangoi et $a{ }^{11}$ Long et $a l^{10}$ reported positivity in $67 \%$ of primary pancreatic neuroendocrine tumors and in $50 \%$ of metastatic pancreatic neuroendocrine tumors, whereas Sangoi et a ${ }^{11}$ reported positivity in $74 \%$ of primary pancreatic neuroendocrine tumors and in $65 \%$ of metastatic pancreatic neuroendocrine tumors. These values are relatively comparable to 
Table 6 Comparison of Islet 1 and PAX8 immunohistochemistry in well-differentiated neuroendocrine tumors with previously published studies

\begin{tabular}{|c|c|c|c|c|c|}
\hline \multirow[t]{2}{*}{ Site } & \multicolumn{2}{|c|}{ Islet 1, total number pos (\%) } & \multicolumn{3}{|c|}{ PAX8, total number pos (\%) } \\
\hline & Schmitt et al ${ }^{12}$ & Koo et al (this issue) & Long et $\mathrm{al}^{10}$ & Sangoi et al ${ }^{11}$ & Koo et al (this issue) \\
\hline Pancreas & $58 / 84(69)$ & $27 / 33(82)$ & $42 / 63(67)$ & 49/66 (74) & 29/33 (88) \\
\hline Pulmonary & $5 / 31(16)$ & $2 / 31(6)$ & $0 / 20$ & $0 / 21$ & $7 / 31(23)$ \\
\hline Ileum & $1 / 30(3)$ & $0 / 23$ & $0 / 31$ & $0 / 31$ & $0 / 23$ \\
\hline Rectum & $0 / 1$ & 12/14 (86) & 11/13 (85) & $5 / 17(29)$ & 11/14 (79) \\
\hline Stomach & $4 / 20(20)$ & $0 / 9$ & $1 / 5(20)$ & $2 / 20(10)$ & $2 / 9(22)$ \\
\hline \multicolumn{6}{|c|}{ Liver metastases } \\
\hline Pancreas & $12 / 18(67)$ & $19 / 28(68)$ & 9/18 (50) & 20/31 (65) & $15 / 28(54)$ \\
\hline Pulmonary & 0 & $0 / 5$ & 0 & $0 / 2$ & $0 / 5$ \\
\hline Ileum & $0 / 17$ & $0 / 37$ & $0 / 16$ & $0 / 11$ & $0 / 37$ \\
\hline Rectum & 0 & $1 / 1(100)$ & 0 & $0 / 1$ & $0 / 1$ \\
\hline Colon & $0 / 2$ & $0 / 1$ & 0 & 0 & $0 / 1$ \\
\hline
\end{tabular}

our findings with PAX8 in primary and metastatic pancreatic neuroendocrine tumors (88 and 54\%, respectively), though our observed rate of positivity for PAX8 in primary pancreatic neuroendocrine tumors is somewhat higher than in the prior two studies. Similar to our study, both prior studies also reported PAX8 positivity in a subset of primary gastric neuroendocrine tumors, in a number of primary rectal neuroendocrine tumors, and in none of the primary or metastatic ileal neuroendocrine tumors. Although Sangoi et $a l^{11}$ reported mostly weak staining with PAX8 in primary rectal neuroendocrine tumors (4/5), we observed mostly moderate to strong staining. In contrast to both studies, we observed a subset of primary pulmonary neuroendocrine tumors with positive nuclear PAX8 staining $(23 \%, 7 / 31)$.

In the final stages of preparation of this manuscript, a letter to the editor by Moreno et $\mathrm{al}^{22}$ was published in the American Journal of Surgical Pathology, questioning the specificity of the polyclonal PAX8 antibody (Proteintech) used in studies by Long et $a l^{10}$ and Sangoi et $a l^{11}$ and which was also used in the current study. Moreno et $a l^{22}$ assert that the Proteintech PAX8 polyclonal antibody displays cross-reactivity with PAX6 and that PAX8 is likely not an accurate biomarker for pancreatic neuroendocrine tumors. Although this is certainly a possibility, our study nevertheless conclusively demonstrates that the Proteintech polyclonal PAX8 antibody reliably differentiates pancreatic from ileal neuroendocrine tumors.

Several previous studies evaluating TTF1 as a marker for pulmonary neuroendocrine tumors and CDX2 as a marker for gastrointestinal neuroendocrine tumors have shown variable sensitivity and specificity with these immunohistochemical stains. Oliveira et $\mathrm{al}^{7}$ first reported a sensitivity of $95 \%$ (19/20) with TTF1 as a marker for pulmonary neuroendocrine tumors, but subsequent studies have shown sensitivities with TTF1 ranging from 28 to
$53 \% .^{3,6,8,9,12}$ TTF1 has consistently been shown to be highly specific for pulmonary neuroendocrine tumors, although rare cases of TTF1 positivity in a pancreatic neuroendocrine tumor and an ileal neuroendocrine tumor were reported by Schmitt et al. ${ }^{12}$ Overall, our study reiterates the previous findings of high specificity but suboptimal sensitivity of TTF1 for pulmonary neuroendocrine tumors.

In gastrointestinal neuroendocrine tumors, CDX2 has been found to be positive more often in midgut tumors than in foregut or hindgut tumors. ${ }^{5,8}$ Consistently positive in ileal and appendiceal neuroendocrine tumors, CDX2 has also been variably positive in rectal, large intestinal, gastric, and duodenal neuroendocrine tumors. ${ }^{2,4-6,8,9,12}$ CDX2 positivity in pancreatic neuroendocrine tumors has been described ${ }^{2,4,8,12}$ and has also been reported rarely in pulmonary neuroendocrine tumors. ${ }^{4}$ In our study, we only found positive CDX2 staining in one metastatic pancreatic neuroendocrine tumor, which showed co-expression with Islet 1.

This study represents the largest number of metastatic neuroendocrine tumors in the liver studied to date for expression of Islet 1 or PAX8 by immunohistochemistry. Overall, our findings support the contention that both Islet 1 and PAX8 are reliable immunohistochemical markers for neuroendocrine tumors of pancreatic origin and would be useful adjuncts to other markers (TTF1, CDX2) already currently used in the workup of a metastatic neuroendocrine tumor of unknown primary. Importantly, in our study, positive immunoreactivity for Islet 1 or PAX8 clearly differentiated between pancreatic and ileal neuroendocrine tumors, which are the two main diagnostic considerations in a metastatic neuroendocrine tumor of unknown primary involving the liver. Although we found immunoreactivity to Islet 1 and PAX8 in some primary pulmonary neuroendocrine tumors and in many rectal neuroendocrine tumors, these are often not major diagnostic considerations in the workup of a metastatic 
neuroendocrine tumor of unknown primary due to the low propensity for pulmonary and rectal neuroendocrine tumors to metastasize to the liver. Nevertheless, caution should be used in an Islet 1 or PAX8-positive metastatic neuroendocrine tumor, and definitive exclusion of a pulmonary or rectal primary should be made clinically. In summary, we believe that an immunohistochemical panel, including Islet 1, PAX8, TTF1, and CDX2 would be helpful in establishing the site of origin when confronted with a metastatic neuroendocrine tumor of unknown primary.

\section{Acknowledgements}

We thank Fai Chung for immunohistochemistry support. The monoclonal anti-Islet 1 antibody, developed by Thomas M Jessell and Susan Brenner-Morton, was obtained from the Developmental Studies Hybridoma Bank developed under the auspices of the NICHD and maintained by The University of Iowa, Department of Biology, Iowa City, IA 52242, USA.

\section{Disclosure/conflict of interest}

The authors declare no conflict of interest.

\section{References}

1 Modlin IM, Oberg K, Chung DC, et al. Gastroenteropancreatic neuroendocrine tumours. Lancet Oncol 2008;9:61-72.

2 Barbareschi M, Roldo C, Zamboni G, et al. CDX-2 homeobox gene product expression in neuroendocrine tumors: its role as a marker of intestinal neuroendocrine tumors. Am J Surg Pathol 2004;28:1169-1176.

3 Du EZ, Goldstraw P, Zacharias J, et al. TTF1 expression is specific for lung primary in typical and atypical carcinoids: TTF1-positive carcinoids are predominantly in peripheral location. Hum Pathol 2004;35: 825-831.

4 Erickson LA, Papouchado B, Dimashkieh H, et al. Cdx2 as a marker for neuroendocrine tumors of unknown primary sites. Endocr Pathol 2004;15:247-252.

5 Jaffee IM, Rahmani M, Singhal MG, et al. Expression of the intestinal transcription factor CDX2 in carcinoid tumors is a marker of midgut origin. Arch Pathol Lab Med 2006;130:1522-1526.

6 Lin X, Saad RS, Luckasevic TM, et al. Diagnostic value of CDX-2 and TTF1 expressions in separating metastatic neuroendocrine neoplasms of unknown origin. Appl Immunohistochem Mol Morphol 2007;15: 407-414.

7 Oliveira AM, Tazelaar HD, Myers JL, et al. Thyroid transcription factor-1 distinguishes metastatic pulmonary from well-differentiated neuroendocrine tumors of other sites. Am J Surg Pathol 2001;25:815-819.
8 Saqi A, Alexis D, Remotti F, et al. Usefulness of CDX2 and TTF1 in differentiating gastrointestinal from pulmonary carcinoids. Am J Clin Pathol 2005;123: 394-404.

9 Srivastava A, Hornick JL. Immunohistochemical staining for CDX-2, PDX-1, NESP-55, and TTF1 can help distinguish gastrointestinal carcinoid tumors from pancreatic endocrine and pulmonary carcinoid tumors. Am J Surg Pathol 2009;33:626-632.

10 Long KB, Srivastava A, Hirsch MS, et al. PAX8 expression in well-differentiated pancreatic endocrine tumors: correlation with clinicopathologic features and comparison with gastrointestinal and pulmonary carcinoid tumors. Am J Surg Pathol 2010;34:723-729.

11 Sangoi AR, Ohgami RS, Pai RK, et al. PAX8 expression reliably distinguishes pancreatic well-differentiated neuroendocrine tumors from ileal and pulmonary well-differentiated neuroendocrine tumors and pancreatic acinar cell carcinoma. Mod Pathol 2011;24: 412-424.

12 Schmitt AM, Riniker F, Anlauf M, et al. Islet 1 (Isl1) expression is a reliable marker for pancreatic endocrine tumors and their metastases. Am J Surg Pathol 2008;32:420-425.

13 Rindi G, Arnold R, Bosman FT, et al. Nomenclature and classification of neuroendocrine neoplasms of the digestive system. In: Bosman FT, Carneiro F, Hruban $\mathrm{RH}$, Theise ND (eds). WHO Classification of Tumors of the Digestive System. IARC Press, Lyon, 2010, pp 13-14.

14 Beasley MB, Thunnissen FB, Hasleton PS, et al. Carcinoid tumor. In: Travis WD, Brambilla E, MullerHermelink HK, Harris CC (eds). Pathology and Genetics of Tumours of the Lung, Pleura, Thymus and Heart. IARC Press: Lyon, France, 2004, pp 59-62.

15 DeLong ER, DeLong DM, Clarke-Pearson DL. Comparing the areas under two or more correlated receiver operating characteristic curves: a nonparametric approach. Biometrics 1988;44:837-845.

16 Yao JC, Hassan M, Phan A, et al. One hundred years after 'carcinoid': epidemiology of and prognostic factors for neuroendocrine tumors in 35825 cases in the United States. J Clin Oncol 2008;26:3063-3072.

17 Kulke MH, Siu LL, Tepper JE, et al. Future directions in the treatment of neuroendocrine tumors: consensus report of the National Cancer Institute Neuroendocrine Tumor clinical trials planning meeting. J Clin Oncol 2011;29:934-943.

18 Boudreaux JP, Putty B, Frey DJ, et al. Surgical treatment of advanced-stage carcinoid tumors: lessons learned. Ann Surg 2005;241:839-845.

19 Givi B, Pommier SJ, Thompson AK, et al. Operative resection of primary carcinoid neoplasms in patients with liver metastases yields significantly better survival. Surgery 2006;140:891-897.

20 Hobert O, Westphal H. Functions of LIM-homeobox genes. Trends Genet 2000;16:75-83.

21 Lang D, Powell SK, Plummer RS, et al. PAX genes: roles in development, pathophysiology, and cancer. Biochem Pharmacol 2007;73:1-14.

22 Moreno CM, Lorenzo PI, Delgado I, et al. Pax8 detection in well-differentiated pancreatic endocrine tumors: how reliable is it? Am J Surg Pathol 2011;35: 1906-1908. 